\title{
Comparison between 2.5D and 3D simulations of coronal mass ejections
}

\author{
C. Jacobs, B. van der Holst, and S. Poedts
}

Centrum voor Plasma-Astrofysica, K.U.Leuven, Celestijnenlaan 200B, 3001 Leuven, Belgium

e-mail: Carla. Jacobs@wis . kuleuven.be

Received 15 February 2007 / Accepted 19 April 2007

\begin{abstract}
Context. The shocks and magnetic clouds related to Coronal Mass Ejections (CMEs) in the solar corona and interplanetary space (IP) play an important role in the study of space weather. In order to study the evolution of these IP shocks, numerical simulations of a simplified CME model were performed.

Aims. In an earlier study, the effect of the background wind on the evolution of interplanetary shock waves was investigated, where the computations were carried out under the assumption of axial symmetry. The assumption of axial symmetry might be a good approach for the solar corona under conditions of solar minimum, but for the study of CMEs this assumption is definitely no longer valid as CMEs possess clearly a fully three dimensional (3D) structure. From this perspective, the previous simulations were repeated, but now in a three dimensional set-up in order to point out the differences between the $2.5 \mathrm{D}$ and $3 \mathrm{D}$ simulations and to check the quality and reliability of the $2.5 \mathrm{D}$ simulations.

Methods. The computations were performed in the framework of ideal magnetohydrodynamics (MHD) and to advance the ideal MHD equations in time a parallel finite volume code with explicit upwind solver was used. The shock waves are generated in a similar way in both the 3D and 2.5D simulations, namely by a simple density-blob model. The 3D and 2.5D simulations are all performed with the same numerical methods and on comparable grids, such that the differences between the simulations are purely due to the dimensionality of the problem, and/or the initial parameters for the CME generation.

Results. Three different axisymmetric simulations of CME propagation are compared with the fully three dimensional computation. The 2.5D simulations differ from each other in the parameters used for CME initiation. In a first simulation, the same initial parameters as for the 3D case were taken, in a second simulation the initial amount of mass in the 2.5D and 3D CME was the same, and in a third simulation they had a comparable amount of momentum. It turned out that the latter one compared best with the 3D results. Conclusions. As $2.5 \mathrm{D}$ computations are computationally much cheaper than $3 \mathrm{D}$ computations, we conclude that the $2.5 \mathrm{D}$ simulations of the CME evolution are a good first approach and resemble well the 3D result, provided that the appropriate initiation parameters are chosen.
\end{abstract}

Key words. Sun: coronal mass ejections (CMEs) - magnetohydrodynamics (MHD) - methods: numerical

\section{Introduction}

Coronal Mass Ejections (CMEs) belong to the most violent and fascinating phenomena in the solar system. During these dramatic eruptions enormous amounts of solar material (in the range of $10^{13}-10^{16} \mathrm{~g}$ ) are ejected into interplanetary space on a timescale of only a few hours. Typical CME velocities are in the range of $400-500 \mathrm{~km} \mathrm{~s}^{-1}$, but can amount to more than $2000 \mathrm{~km} \mathrm{~s}^{-1}$. These events involve large-scale changes in the coronal structure and significant disturbances in the solar wind. Especially the massive, fast CMEs are interesting to study as they cause shock waves that propagate through the heliosphere, which can accelerate energetic particles and give rise to so-called gradual Solar Energetic Particle (SEP) events. The interplanetary (IP) shocks, energetic particles and magnetic clouds created by CMEs can interact with the magnetosphere of the Earth, causing geo-magnetic storms which can have harmful consequences for communication and navigation systems, power supplies, etc., which promotes CMEs to one of the most important solar drivers of space weather. Consequently, a careful study of the origin, the structure, and the propagation characteristics of these violent solar phenomena is essential for a deeper insight into space weather physics. This, in turn, is required for more accurate and reliable predictions and long-term forecasts of the space weather.

Numerical modelling of solar transients and prediction of space weather is a challenging task and asks for a large amount of computational power and the continuous improvement of numerical techniques and physical models. During the last few years significant progress has been made in the field of computational magneto-fluid-dynamics applied to solar related phenomena. Riley et al. (2001, 2002), for example, developed an empirically driven MHD model for the solar corona and inner heliosphere and investigated the evolution of the heliospheric current sheet during the course of the solar cycle. Roussev et al. (2003) constructed a three-dimensional model of the solar wind incorporating solar magnetogram observations. In this model the solar wind is powered by the energy interchange between the coronal plasma and large-scale MHD turbulence. Detman et al. (2006) are developing the Hybrid Heliospheric Modeling System. It combines a source surface (potential field) 
current sheet model for the solar corona and a time dependent 3D MHD solar wind model to predict the solar wind conditions at Earth. An empirical relationship between the magnetic flux tube expansion factor and the solar wind speed at 0.1 AU (Wang \& Sheeley 1990) is a crucial element in the model. Odstrcil et al. $(2004,2005)$ numerically simulated the 12 May 1997 CME event and tried to reproduce the plasma parameters near Earth. The Space Weather Modelling Framework (Tóth et al. 2005), recently developed at the university of Michigan, has as purpose to model a CME event from Sun to the Earth, from initiation up to interaction with the magnetosphere of the Earth. One has the choice between different models for the solar background wind as well as different CME initiation models. Even if the physical models can be improved such that solar wind models and CME models become more realistic, running the simulations faster than real time remains a challenging technical task as high grid resolutions, coupling of different physical regimes and massive parallel computations are indispensable.

In earlier work (Jacobs et al. 2005; Chané et al. 2005) the influence of the background solar wind on the CME evolution was investigated and we came to the conclusion that the applied model for the background wind has an effect on the propagation speed, the shock strength, the spread angle and the mass distribution in the CME. The models used for the background solar wind were reconstructions of three popular models often described in literature and all of them were axisymmetric. The assumption of axial symmetry makes sense for solar minimum when the global magnetic field of the Sun is more or less a dipole and simplifies the computations significantly. However, in real life CMEs possess clearly a fully three-dimensional structure. In spite of the increased computer power, 3D computations are time and memory demanding. In the present report, we compare the results of a 3D simulation with similar simulations in $2.5 \mathrm{D}$ and we point out the differences in order to check the reliability of the $2.5 \mathrm{D}$ models.

In the next section, the numerical technique and the applied model for the solar wind and CME triggering will be described briefly. In Sect. 3, the results of the 3D simulation are discussed and the corresponding axisymmetric simulations are described and analysed. The last section contains our conclusions.

\section{Numerical model}

The ideal MHD equations are solved by means of a finite volume code derived from the Versatile Advection Code (VAC) (Tóth 1996). This code supports parallel computations on distributed memory machines, using the Message Passing Interface (MPI). For the numerical scheme we opted for the Lax-Friedrichs scheme, as this scheme is stable and robust. The magnetic field is kept divergence free by using the vector potential on the nodal points instead of the cell-centred magnetic field components, as suggested by Balsara \& Spicer (1999). The equations are solved in a spherical geometry ( $r, \theta, \phi$-coordinates) where the computational domain is restricted to $1-30 R_{\odot}$ in the radial direction and covers the region between north and south pole of the Sun, i.e. $0 \leq \theta \leq \pi$. For the 3D simulation a full sphere is modeled where the cells are equidistant in the longitudinal direction $(0 \leq \varphi \leq 2 \pi)$. The grid resolution was taken to be $644 \times 95 \times$ 184 , including 2 ghostcells at each end. The grid shows an accumulation of cells towards the solar surface and towards the equator, where the grid size varies from $\Delta r=0.005 R_{\odot}$ near the solar base to $\Delta r=0.16 R_{\odot}$ at the outer boundary and from $\Delta \theta=4^{\circ}$ at the poles to $\Delta \theta=0.8^{\circ}$ at the equator. For the $2.5 \mathrm{D}$ simulations the grid is limited to a meridional plane of the $3 \mathrm{D}$ mesh.
The high resolution in the radial direction was needed in order to capture the CME shock structure satisfactorily.

\subsection{Solar wind}

The background solar wind is similar to the model first developed by Groth et al. (2000) and described in more detail by Manchester et al. (2004). The full set of MHD equations is solved in a corotating frame along with an extra added gravitational force as well as an additional heating term that mimics the radiative losses, thermal conduction and other heating mechanisms. The initial condition is the hydrodynamic solar wind solution of Parker (1958), with an added magnetic dipole field with a field strength of $2.2 \mathrm{G}$ at the poles. This differs from Manchester et al. (2004), who used a mixed dipole and octupole field, having a dependence of the azimuthal direction. Since we want to compare a 3D simulation with similar axisymmetric simulations, we restricted the magnetic field to a dipole configuration, showing no dependence of the azimuthal direction. At the solar base, the density is fixed to $\rho_{*}=1.67 \times 10^{-16} \mathrm{~g} \mathrm{~cm}^{-3}$, the temperature to $T_{*}=1.5 \times 10^{6} \mathrm{~K}$, and the angular velocity to $\Omega_{*}=2.8 \times 10^{-6} \mathrm{rad} \mathrm{s}^{-1}$. The boundary conditions on the vector potential $\mathbf{A}$ are chosen so that the radial component of the magnetic field remains the same as that of the initial dipole. All other variables at the base are extrapolated. At the outer boundary the plasma flow is superfast and outflow boundary conditions are applied. As the initial solution relaxes to the steady state, the original dipole configuration of the magnetic field is lost and regions of open and closed field lines develop. The additional heating term $Q$ in the energy equation creates the regions of fast and slow wind and takes the form:

$Q=\rho q_{0}\left(T_{0}-\gamma \frac{p}{\rho}\right) \exp ^{-\frac{\left(r-R_{\odot}\right)^{2}}{\sigma_{0}^{2}}}$

where $q_{0}$ has a value of $q_{0}=1.2 \times 10^{6} \mathrm{erg} \mathrm{g}^{-1} \mathrm{~K}^{-1} \mathrm{~s}^{-1}$, and both $T_{0}$ and $\sigma_{0}$ have an $r$ and $\theta$ dependence. The target temperature has a value of $T_{0}=2.5 \times 10^{6} \mathrm{~K}$ in the region towards the equator below a critical angle $\theta_{0}(r)$, and a value of $T_{0}=4.375 \times$ $10^{6} \mathrm{~K}$ poleward of the critical angle. The heating scale height $\sigma_{0}$ takes a value of $4.5 R_{\odot}$ equatorward of the critical angle, and increases to a value of $9 R_{\odot}$ at the poles. At the solar base the critical angle has a value of $\theta_{0}\left(r=1 R_{\odot}\right)=18^{\circ}$ and the radial dependence of $\theta_{0}$ is the same as described in Manchester et al. (2004). This wind model shows no dependence of the azimuthal direction and is an approximation for the solar minimum.

\section{2. $C M E$ generation}

As we are primarily interested in the shocks associated with CMEs and not in the initiation of the CMEs itself, a simple "density-driven" model is used to trigger the CMEs. Hence, the shocks are generated by launching a high-density plasma blob on the wind at a certain speed $v_{\mathrm{cme}}$ in a prescribed radial direction $\theta_{\text {cme }}$. The velocity and density profile in the initial disturbance both take the form:

$f=\frac{f_{\mathrm{cme}}}{2}\left(1-\cos \pi \frac{d_{\mathrm{cme}}-d}{d_{\mathrm{cme}}}\right)$,

where $f$ indicates the added density $\rho$ or radial velocity $v_{r}, f_{\text {cme }}$ is the maximum density $\rho_{\text {cme }}$ or radial velocity $v_{\text {cme }}$ in the plasma bubble, $d_{\text {cme }}$ is the radius of the bubble, and $d$ is the distance to the centre of the blob. See Jacobs et al. (2005) for a description of this model. The centre of the plasma blob is initially at 

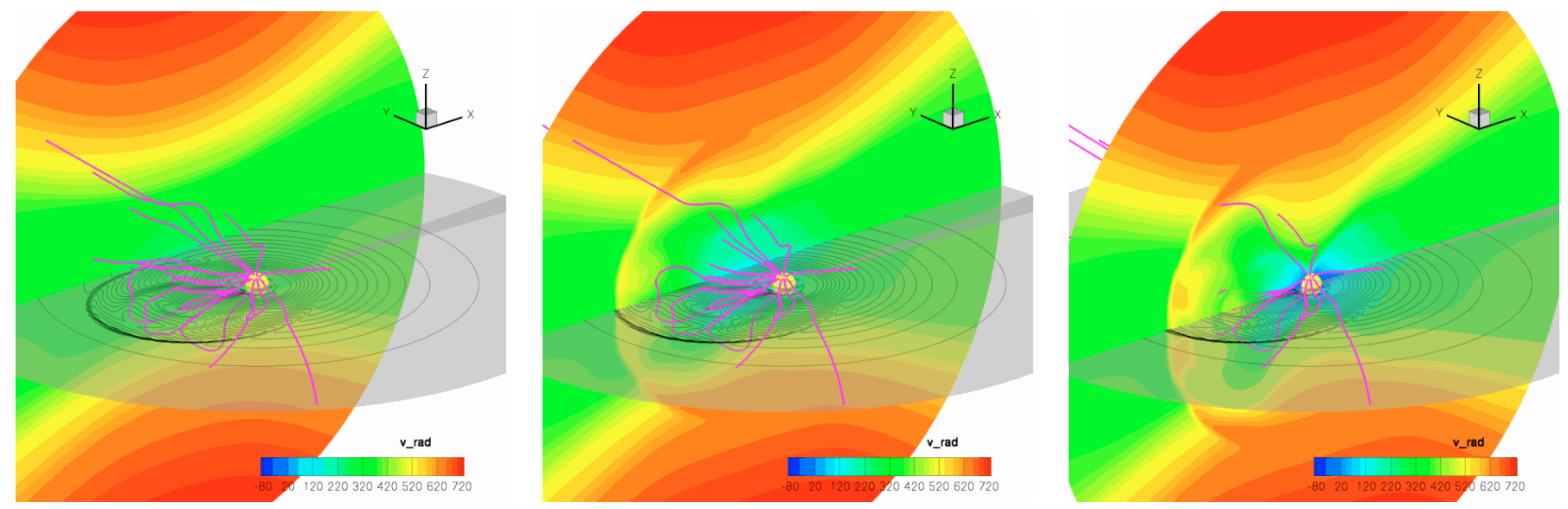

Fig. 1. Snapshot of the 3D simulation after $t=3 \mathrm{~h} 50 \mathrm{~min}$. Shown are contours for the radial velocity in the equatorial plane and in three different slices parallel with the $X Z$-plane. Pink lines indicate magnetic field lines.
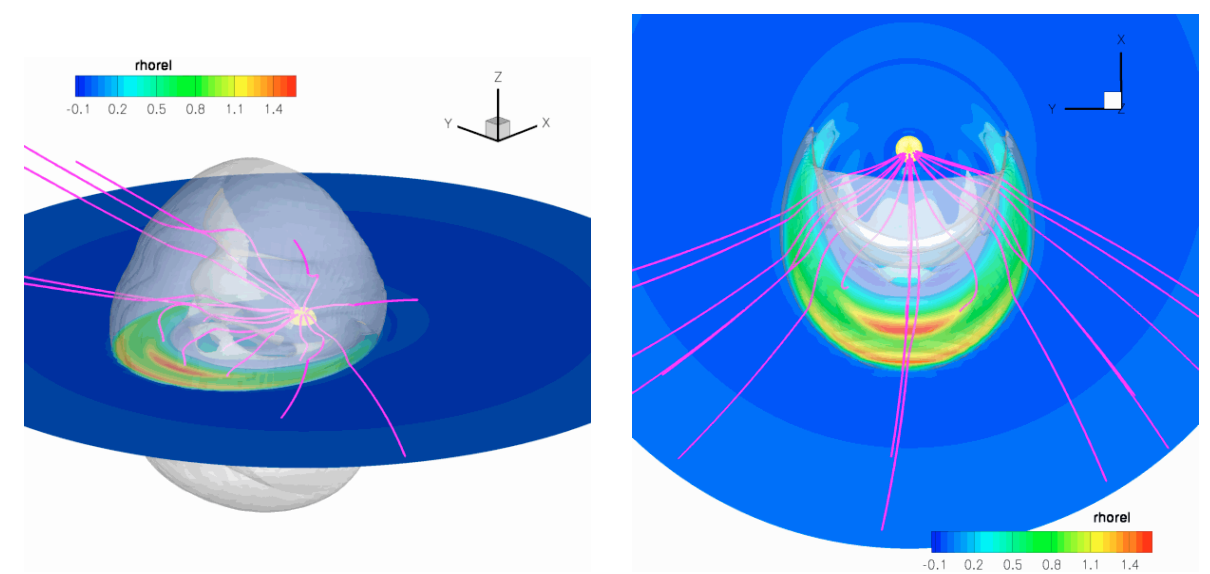

Fig. 2. Two different points of view of a snapshot of the 3D simulation after $t=3 \mathrm{~h} 50 \mathrm{~min}$. Shown are contours for the relative density $\bar{\rho}$ in the equatorial plane and an isosurface where $\bar{\rho}=0.1$. Pink lines indicate magnetic field lines.

a certain radial position $r_{\text {cme }}$ and latitude $\theta_{\text {cme }}$. For the simulations shown here $r_{\text {cme }}$ was taken $1.5 R_{\odot}, d_{\text {cme }}$ equals $0.29 R_{\odot}$, and the blob was launched in the equatorial plane $\left(\theta_{\mathrm{cme}}=0^{\circ}\right)$. In case of the $3 \mathrm{D}$ simulation, a third parameter is needed to fix the initial position of the plasma blob, namely $\varphi_{\text {cme }}$, indicating the longitudinal position of the blob-centre. This parameter is taken equal to $\varphi_{\text {cme }}=\pi$. The values taken for $r_{\text {cme }}, \theta_{\text {cme }}$, and $\varphi_{\text {cme }}$ correspond with launching the plasma blob along the negative $X$-axis. After the initiation, the CME was followed during approximately $7 \mathrm{~h}$. To reach this time, about 50000 iterations were needed in the fully three dimensional computation, giving a total simulation time of about $47 \mathrm{~h}$ on $128 \mathrm{CPUs}$ of the VIC-cluster at the K. U. Leuven. In comparison: the axisymmetric computations needed only around 13000 iterations (because of the larger time step) and were finished in less than 10 min when 32 CPUs were used.

\section{Results}

In order to create fast shocks the maximum radial velocity in the blob $v_{\text {cme }}$ is about $1000 \mathrm{~km} \mathrm{~s}^{-1}$ for every simulation. In the case of the 3D simulation a maximum in density of $\rho_{\mathrm{cme}}=10 \rho_{*}$ is taken, i.e. the density in the centre of the blob is taken 10 times higher than the density at the solar base $\rho_{*}$. This value for $\rho_{\text {cme }}$ corresponds to a total mass in the blob of $\approx 10^{16} \mathrm{~g}$, which is a realistic amount of mass for a CME. Plots of a snapshot of this simulation are shown in Figs. 1 and 2. In Fig. 1, contours of the radial velocity are shown for different slices parallel with the $X Z$-plane. From this figure it is clearly seen that the background wind possesses regions of fast wind, where the maximum wind speed is more than $700 \mathrm{~km} \mathrm{~s}^{-1}$, and regions of slow wind. In Fig. 2, contours of the relative density $\bar{\rho}$ are shown. The relative density expresses the excess or depletion in density compared to the original steady state background wind. The white transparent isosurface corresponds to the position where the relative density has a value of $\bar{\rho}=0.1$, i.e. where the density in the simulation is $10 \%$ higher than the density of the background wind, indicating the spatial boundaries of the CME. Figure 3 shows a plot of the position of the centre of relative mass during the time evolution of the $3 \mathrm{D}$ plasma blob. The centre of relative mass $\boldsymbol{r}_{\mathrm{CRM}}$ is defined as

$\boldsymbol{r}_{\mathrm{CRM}}=\frac{\int_{V} \boldsymbol{r} \bar{\rho}(\boldsymbol{r}) \mathrm{d}^{3} \boldsymbol{r}}{\int_{V} \bar{\rho}(\boldsymbol{r}) \mathrm{d}^{3} \boldsymbol{r}}$.

Because of the up-down symmetry of the simulation, the centre of mass is positioned in the equatorial plane ( $X Y$-plane). From the plot it can be seen how the rotation of the Sun influences the propagation of the CME, as expected, however, as the simulation domain is restricted till $30 R_{\odot}$ this effect is only minor. The effect of solar rotation can also be seen in the magnetic field line traces shown in pink in Figs. 1 and 2. 


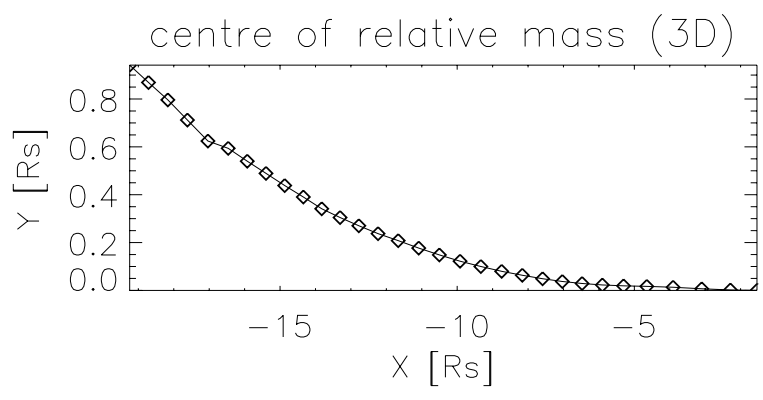

Fig. 3. Position of the centre of relative mass in the equatorial plane. The centre of mass deflects from the $X$-axis due to the rotation of the Sun.

The 3D simulation is compared with $2.5 \mathrm{D}$ simulations with 3 different values for $\rho_{\mathrm{cme}}$. The first axisymmetric simulation has $\rho_{\text {cme }}=10$, just like the $3 \mathrm{D}$ simulation, but in the axisymmetric case the initial plasma blob corresponds to a torus of higher density around the Sun, and so the total amount of mass in the torus is much higher than the total amount of mass in the 3D plasma blob, namely: $4 \times 10^{17} \mathrm{~g}$, which is higher than the observational values. The second $2.5 \mathrm{D}$ simulation has $\rho_{\mathrm{cme}}=0.27$. With this value for $\rho_{\text {cme }}$ the total amount of mass in the torus is the same as for the 3D plasma blob. Of course, it is not only the amount of mass in the blob that is important for the CME evolution, but also the initial momentum. In a third axisymmetric simulation, the parameters $d_{\text {cme }}$ and $\rho_{\text {cme }}$ are adapted in such a way that, if only a part of the torus would be considered, that has the same width as the 3D plasma bubble, then this part of the torus has the same initial volume and the same amount of initial momentum in the radial direction as the $3 \mathrm{D}$ plasma blob. This gives a value for the blob radius of $d_{\mathrm{cme}}=0.238 R_{\odot}$ and a value of $\rho_{\text {cme }}=2.4$ for the peak in density. Figure 4 shows a snapshot of the relative density and the radial velocity for the three axisymmetric simulations and for the full three dimensional case. In order to be able to compare the $2.5 \mathrm{D}$ and $3 \mathrm{D}$ results, the $3 \mathrm{D}$ results are shown in the meridional plane that initially contained the centre of the plasma blob, i.e. the $X Z$-plane. As the effect of solar rotation is small, the CME will be more or less symmetric around this plane. In Fig. 5 cuts for the density and radial velocity are shown in the equatorial plane, along the $X$-axis. From these figures it is seen that the $2.5 \mathrm{D}$ run having the same momentum $\left(d_{\mathrm{cme}}=0.24\right.$ and $\left.\rho_{\mathrm{cme}}=2.4\right)$ corresponds the best with the $3 \mathrm{D}$ simulation.

In order to quantify the differences in propagation speed between the different simulations, Fig. 6 shows the position of the CME front in the $X Z$-plane at different moments. As expected (since the initial velocity is always the same), the most massive CME (case $\rho_{\text {cme }}=10$ ) propagates the fastest and the low density $\mathrm{CME}$ (case $\rho_{\text {cme }}=0.27$ ) the slowest, while the 2.5D simulation with the same amount of initial momentum propagates more or less at the same speed as the $3 \mathrm{D}$ case. Heighttime plots of the position of the CME front along the equatorial plane are shown in Fig. 7. Sheeley et al. (1999) investigated the height-time curves of several CME events and concluded that fast CMEs show clear evidence of deceleration. The observed height-time curves were fitted with the function:

$r(t)=r_{0}+v_{1} t+\left(1-\mathrm{e}^{-t / \tau}\right)\left(v_{0}-v_{1}\right) \tau$.

The corresponding velocity profile is then given by:

$v(t)=v_{1}+\left(v_{0}-v_{1}\right) \mathrm{e}^{-t / \tau}$,

with $v_{0}$ the initial speed of the CME, and $v_{1}$ the final speed, reached asymptotically in an $e$-folding time $\tau$. This fitting

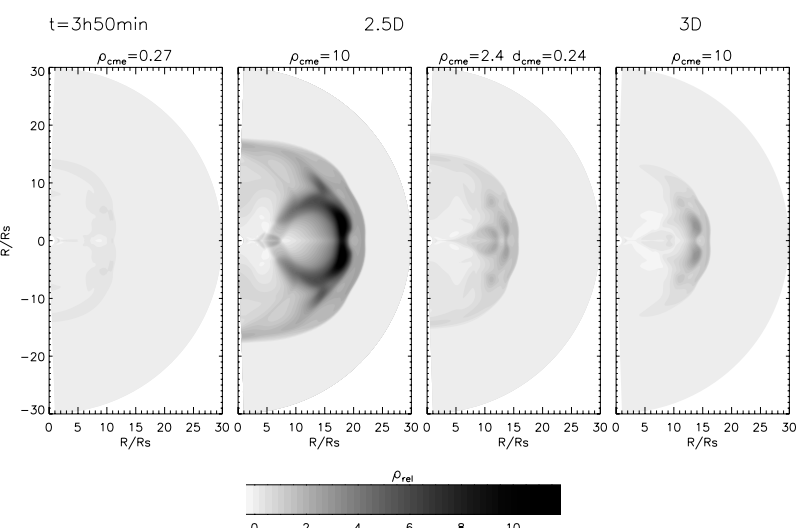

(a) relative density

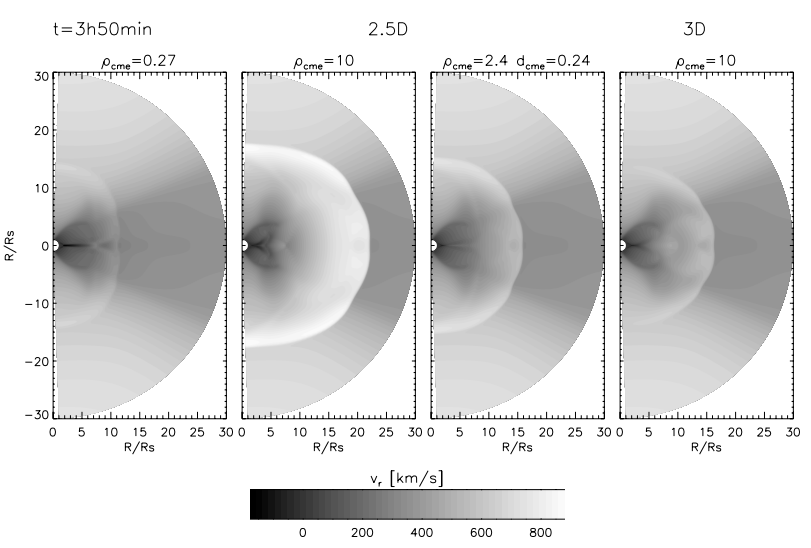

(b) radial velocity

Fig. 4. Contour plots of a) the relative density and b) the radial velocity in the different simulations, 3h50min after the onset of the CME initiation. The frame on the outer right shows the result for the 3D run.

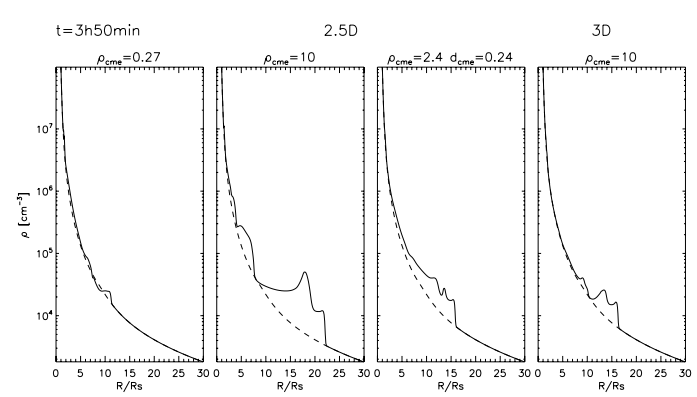

(a) density

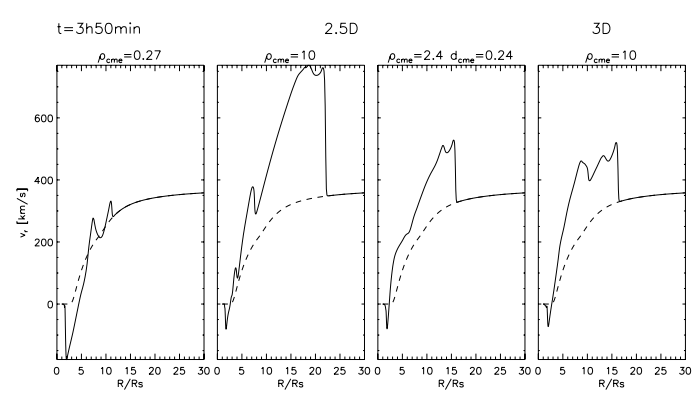

(b) radial velocity

Fig. 5. Cuts along the equator for a) the density and b) the radial velocity. The dashed line indicates the value in the undisturbed background wind. 


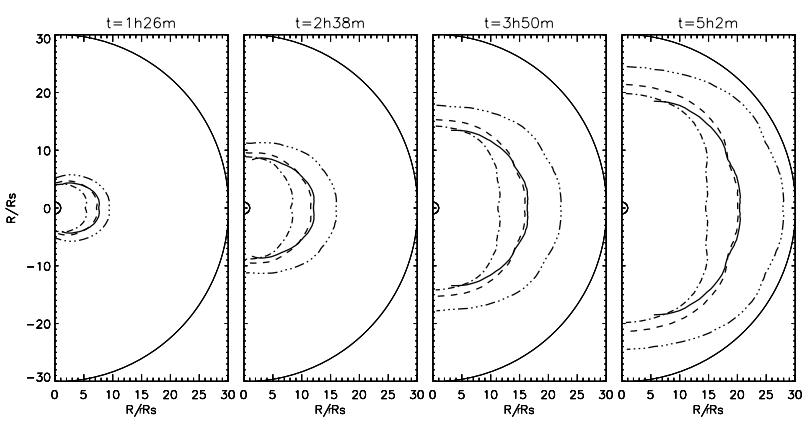

Fig. 6. The position of the CME front at different times. Dash-dot line: $2.5 \mathrm{D}$ and $\rho_{\mathrm{cme}}=0.27$, dash-dot-dot-dot line: $2.5 \mathrm{D}$ and $\rho_{\mathrm{cme}}=10$, dashed line: $2.5 \mathrm{D}$ and $\rho_{\mathrm{cme}}=2.4$, solid line: $3 \mathrm{D}$.

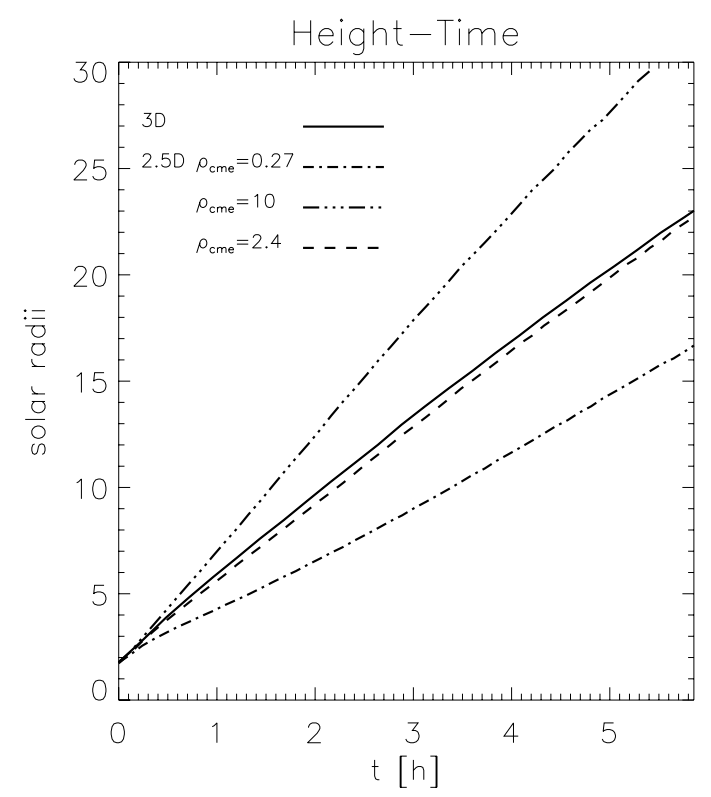

Fig. 7. The position of the CME front along the equator versus time. Dash-dot line: $2.5 \mathrm{D}$ and $\rho_{\mathrm{cme}}=0.27$, dash-dot-dot-dot: $2.5 \mathrm{D}$ and $\rho_{\mathrm{cme}}=$ 10, dashed line: $2.5 \mathrm{D}$ and $\rho_{\mathrm{cme}}=2.4$, solid line: $3 \mathrm{D}$.

technique is applied to the $3 \mathrm{D}$ simulation on both the heighttime plot of the CME front, as on the height-time curve of the centre of relative mass. For the CME front, we find values of $r_{0}=1.65 R_{\odot}, v_{0}=948 \mathrm{~km} \mathrm{~s}^{-1}, v_{1}=647 \mathrm{~km} \mathrm{~s}^{-1}$, and $\tau=1.14 \mathrm{~h}$. The centre of relative mass moves slower with an initial velocity $v_{0}=666 \mathrm{~km} \mathrm{~s}^{-1}$, and asymptotic speed $v_{1}=434 \mathrm{~km} \mathrm{~s}^{-1}$. Values for $r_{0}$ and $\tau$ are $1.57 R_{\odot}$ and $1.28 \mathrm{~h}$ respectively. The heighttime curves and corresponding fits with Eq. (1) are shown in Fig. 8a. The bottom panel, Fig. 8b, shows the velocity versus height as derived from the height-time profiles. Both the front of the CME and the centre of relative mass show a decreasing velocity profile, corresponding to the findings of Sheeley et al. (1999) for fast CME events. The empirical result of Sheeley et al. (1999) was also reproduced by Manchester et al. (2004) in their 3D MHD simulation of a flux rope driven CME.

The average velocity of the CME propagation is given by the slope of the least-square linear fit through the height-time curves, while the average acceleration is obtained by a second order polynomial fit through the height-time curves. These velocities and accelerations are summarised in Table 1 and are comparable with typical observed values for CME acceleration and velocity (Yashiro et al. 2004).

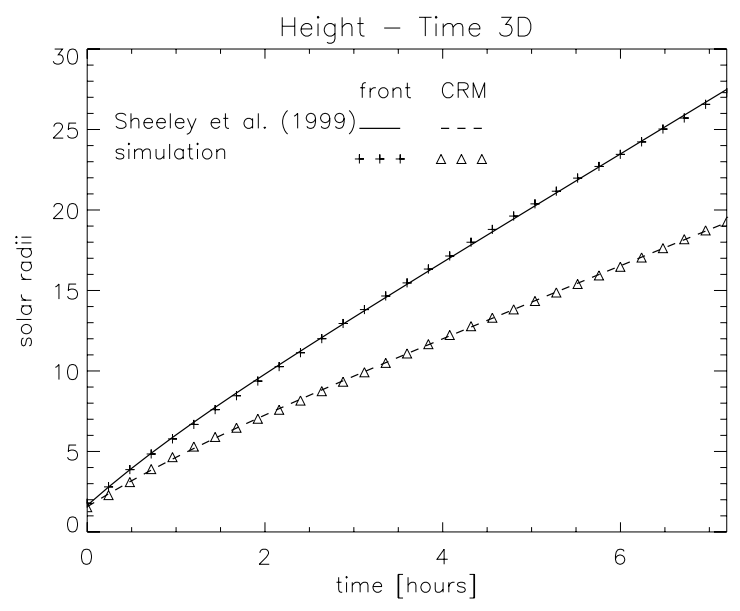

(a) Height-Time plot (3D simulation)

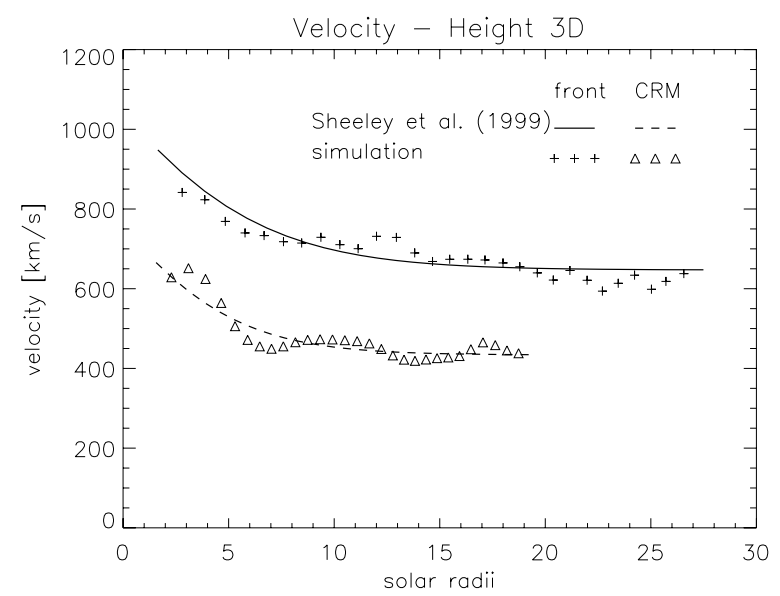

(b) Velocity-Height plot (3D simulation)

Fig. 8. Top: height-time curves for the CME front (+-signs) and the centre of relative mass $(\mathrm{CRM})(\triangle$-signs) along the equator for the $3 \mathrm{D}$ simulation. The fits with Eq. (1) are denoted with a solid line and a dashed line respectively. Bottom: velocity of the front (+-signs) and centre of mass ( $\triangle$-signs $)$ in function of height.

Table 1. Average speed and acceleration of the CME front and of the centre of relative mass (CRM).

\begin{tabular}{cccc|c}
\hline \hline \multicolumn{5}{c}{$2.5 \mathrm{D}$} \\
& $\rho_{\mathrm{cme}}=0.27$ & $\rho_{\mathrm{cme}}=10$ & $\rho_{\mathrm{cme}}=2.4$ & $\rho_{\mathrm{cme}}=10$ \\
\hline$\langle v\rangle$ & $495 \mathrm{~km} \mathrm{~s}^{-1}$ & $1009 \mathrm{~km} \mathrm{~s}^{-1}$ & $680 \mathrm{~km} \mathrm{~s}^{-1}$ & $680 \mathrm{~km} \mathrm{~s}^{-1}$ \\
$\langle a\rangle$ & $4.5 \mathrm{~m} \mathrm{~s}^{-2}$ & $-9.3 \mathrm{~m} \mathrm{~s}^{-2}$ & $-4.3 \mathrm{~m} \mathrm{~s}^{-2}$ & $-7.7 \mathrm{~m} \mathrm{~s}^{-2}$ \\
& & & & \\
$\left\langle v_{\mathrm{CRM}}\right\rangle$ & $383 \mathrm{~km} \mathrm{~s}^{-1}$ & $670 \mathrm{~km} \mathrm{~s}^{-1}$ & $478 \mathrm{~km} \mathrm{~s}^{-1}$ & $463 \mathrm{~km} \mathrm{~s}^{-1}$ \\
$\left\langle a_{\mathrm{CRM}}\right\rangle$ & $8.3 \mathrm{~m} \mathrm{~s}^{-2}$ & $-4.7 \mathrm{~m} \mathrm{~s}^{-2}$ & $-1.5 \mathrm{~m} \mathrm{~s}^{-2}$ & $-5.1 \mathrm{~m} \mathrm{~s}^{-2}$ \\
\hline
\end{tabular}

Vršnak et al. (2004) carried out a statistical study of the kinematics of more than 5000 CMEs measured between 2 and $30 R_{\odot}$. They found a distinct anticorrelation between the average acceleration and the velocity of the CME, that can be represented in the form $a=-k_{1}\left(v-v_{0}\right)$, and interpreted the accelerationvelocity relation in terms of aerodynamic drag. Their reported values for $v_{0}$ are systematically larger than the solar wind speed by some $100-200 \mathrm{~km} \mathrm{~s}^{-1}$. The aerodynamic drag is proportional with the difference between the wind speed and the CME speed: events faster than the solar wind will decelerate, while events slower than the background wind will show a positive 


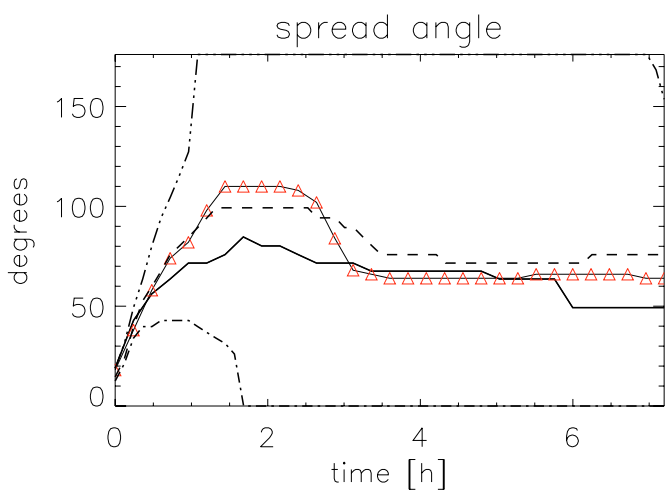

Fig. 9. The time evolution of the spread angle in the $X Z$-plane. Dashdot line: $2.5 \mathrm{D}$ and $\rho_{\mathrm{cme}}=0.27$, dash-dot-dot-dot: $2.5 \mathrm{D}$ and $\rho_{\mathrm{cme}}=10$, dashed line: $2.5 \mathrm{D}$ and $\rho_{\mathrm{cme}}=2.4$, solid line: 3D. The line with the triangles gives the evolution of the spread angle of the 3D simulation in the equatorial plane.

acceleration. Although the average velocity of the slowest simulated event is larger than the wind speed $\left(v_{\text {wind }} \approx 350 \mathrm{~km} \mathrm{~s}^{-1}\right.$ at $30 R_{\odot}$ ), the acceleration is positive, confirming the findings of Vršnak et al. (2004) that in the range between 2 and 30 solar radii a non-negligible propelling force is still acting on the CME. The drag force does not only depend on the solar wind and CME speed, but also on the CME dimensions and density. According to Vršnak et al. (2004) the slope $k_{1}$ of the $a-v$ relation is smaller for wider CMEs. This might explain the difference in acceleration between the 3D simulation and the best corresponding axisymmetric simulation. In Fig. 9 the evolution of the spread angle with time is shown for the different simulations. As criterion for the determination of the spread angle the same as in Jacobs et al. (2005) was taken, i.e. only the part of the CME with a relative density higher than one $(\bar{\rho}>1)$ is considered. We have chosen this criterion since it expresses an excess in density, like indirectly seen in coronagraph images. Whatever level in relative density is chosen in the computation of the spread angle, the spreading of the 3D CME is always less than that of the best corresponding 2.5D simulation. Figure 9 shows that the latitudinal variation of the CME remains more or less constant when travelling through the high corona, which is consistent with observations (St. Cyr et al. 2000). For completeness, also the spreading of the 3D CME in the equatorial plane is shown in Fig. 9.

Although the position of the front of the 2.5D simulation with the same amount of momentum $\left(d_{\mathrm{cme}}=0.238\right.$ and $\rho_{\mathrm{cme}}=$ 2.4) is comparable with the position of the CME front in the 3D simulation, it seems from Figs. 4a and 5a that the distribution of the mass in the CME is slightly different. However, when plotting the radial distance of the relative centre of mass $r_{\mathrm{CRM}}$ in time (see Fig. 10), the curves for the 3D simulation and the axisymmetric simulation with the same amount of momentum almost coincide. Average values of the velocity and acceleration of $r_{\mathrm{CRM}}$ can be found in Table 1. The average acceleration of the centre of relative mass is higher than that of the CME front, meaning that at larger distances the front will get compressed.

\section{Conclusions}

Fully three dimensional time dependent ideal MHD simulations of the evolution of a plasma blob in a steady state background wind were performed and the result was compared with similar simulations that considered an axisymmetric configuration. In the range up to $30 R_{\odot}$ it was found that the $3 \mathrm{D}$ simulation

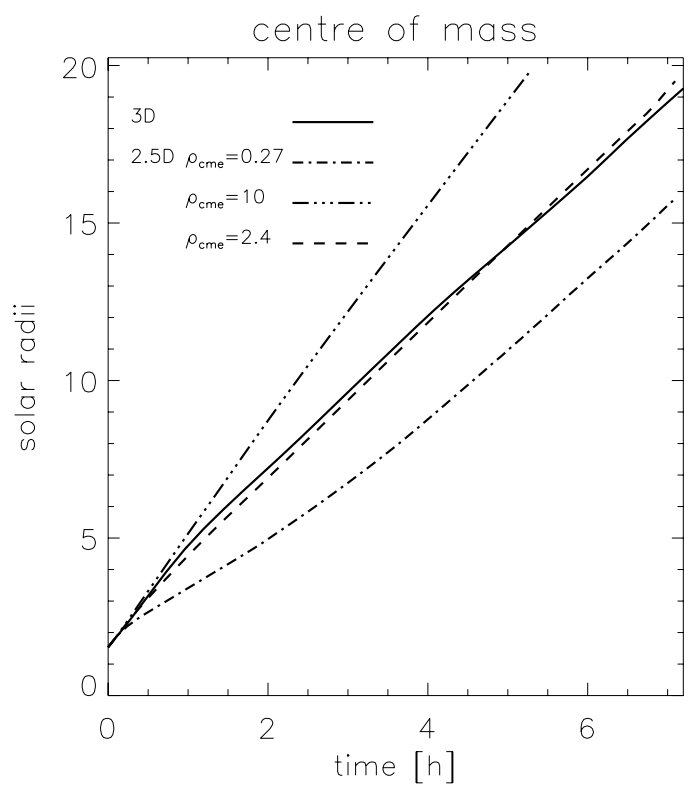

Fig. 10. The position of the centre of relative mass versus time. Dashdot line: $2.5 \mathrm{D}$ and $\rho_{\mathrm{cme}}=0.27$, dash-dot-do-dot line: $2.5 \mathrm{D}$ and $\rho_{\mathrm{cme}}=$ 10 , dashed line: $2.5 \mathrm{D}$ and $\rho_{\mathrm{cme}}=2.4$, solid line: $3 \mathrm{D}$.

was best in agreement with the $2.5 \mathrm{D}$ simulation having parameters $\rho_{\mathrm{cme}}=2.4$ and $d_{\mathrm{cme}}=0.238$, this is corresponding with a simulation having initially the same amount of radial momentum in the plasma blob as the 3D case. For these two simulations the position of the front and of the centre of relative mass showed a similar evolution in time. Because the $2.5 \mathrm{D}$ simulation is spread over a wider area than the 3D simulation, the latter one is subjected to a larger drag force. This would lead to a larger time of arrival when the simulations would be extended up to $1 \mathrm{AU}$. Also, in the axisymmetric simulation the plasmablob corresponds with a torus of high density around the Sun, which is an unrealistic situation. In future work, the results of more realistic 3D CME simulations will be presented, where we will consider a more realistic configuration of the background magnetic field, based on magnetogram data. Recently, our finite volume code is improved with an adaptive mesh refinement technique, making it possible to solve the CME shock structure to higher accuracy. However, even with improved numerical techniques, 3D simulations remain very computational intensive and large parallel systems are needed to perform the computations. The kind of axisymmetric simulations presented here show similar characteristics as observed CMEs and can be used to mimic CME events. For example, by finetuning the initial parameters we were able to fit the velocity, density, and magnetic field profiles of the halo CME event of April 4, 2000 reasonably well (Chané et al. 2006). The axisymmetric simulations can be used as a good first approach to estimate the time of arrival of the shock and the density and velocity structure and correspond well with a fully $3 \mathrm{D}$ simulation, provided that the appropriate initiation parameters are chosen.

Acknowledgements. This research was funded by K.U.Leuven project GOA/2004/01, FWO project G.0304.07 and PRODEX project C90203. The results were obtained on the HPC cluster VIC (K.U. Leuven). With thanks to Dries Kimpe, Jan De Laet, and Wim Obbels. 


\section{References}

Balsara, D. S., \& Spicer, D. S. 1999, J. Comp. Phys., 149, 270

Chané, E., Jacobs, C., van der Holst, B., Poedts, S., \& Kimpe, D. 2005, A\&A, 432, 331

Chané, E., van der Holst, B., Jacobs, C., Poedts, S., \& Kimpe, D. 2006, A\&A, 447, 727

Detman, T., Smith, Z., Dryer, M., et al. 2006, J. Geophys. Res, 111, A07102

Groth, C. P. T., De Zeeuw, D. L., Gombosi, T. I., \& Powell, K. G. 2000, J. Geophys. Res, 105, 25053

Jacobs, C., Poedts, S., van der Holst, B., \& Chané, E. 2005, A\&A, 430, 1099

Manchester, W., Gombosi, T., Roussev, I., et al. 2004, J. Geophys. Res, 109, A01102

Odstrcil, D., Riley, P., \& Zhao, X. P. 2004, J. Geophys. Res, 109, A02116

Odstrcil, D., Pizzo, V. J., \& Arge, C. N. 2005, J. Geophys. Res, 110, A02106
Parker, E. N. 1958, ApJ, 128, 664

Riley, P., Linker, J. A., \& Mikić, Z. 2001, J. Geophys. Res, 106, 15889 Riley, P., Linker, J. A., \& Mikić, Z. 2002, J. Geophys. Res, 107(A7), 1136

Roussev, I. I., Gombosi, T. I., Sokolov, I. V., et al. 2003, ApJ, 595, L57

Sheeley, N. R., Walters, J. H., Wang, Y. M., \& Howard, R. A. 1999, J. Geophys. Res, 104, 24739

St. Cyr, O. C., Howard, R. A., Sheeley, N. R., et al. 2000, J. Geophys. Res, 105, 18169

Tóth, G. 1996, Astrophys. Lett. Comm., 34, 245

Tóth, G., Sokolov, I. V., Gombosi, T. I., et al. 2005, J. Geophys. Res, 110, A12226

Vršnak, B., Ruždjak, D., Sudar, D., \& Gopalswamy, N. 2004, A\&A, 423, 717

Wang, Y.-M., \& Sheeley, Jr., N. R. 1990, ApJ, 355, 726

Yashiro, S., Gopalswamy, N., Michalek, G., et al. 2004, J. Geophys. Res, 109, A07105 